\title{
On the Limit Cycles of a Class of Planar Singular Perturbed Differential Equations
}

\author{
Yuhai Wu and Jingjing Zhou \\ Department of Mathematics, Jiangsu University, Zhenjiang 212013, China \\ Correspondence should be addressed to Yuhai Wu; yuhaiwu@ujs.edu.cn
}

Received 7 December 2013; Accepted 10 February 2014; Published 31 March 2014

Academic Editors: M. Han, Z. Jin, and Y. Xia

Copyright (C) 2014 Y. Wu and J. Zhou. This is an open access article distributed under the Creative Commons Attribution License, which permits unrestricted use, distribution, and reproduction in any medium, provided the original work is properly cited.

\begin{abstract}
Relaxation oscillations of two-dimensional planar singular perturbed systems with a layer equation exhibiting canard cycles are studied. The canard cycles under consideration contain two turning points and two jump points. We suppose that there exist three parameters permitting generic breaking at both the turning points and the connecting fast orbit. The conditions of one (resp., two, three) relaxation oscillation near the canard cycles are given by studying a map from the space of phase parameters to the space of breaking parameters.
\end{abstract}

\section{Introduction}

As we know, the second part of Hilbert's 16th Problem is related with the number and distributions of limit cycles of a general polynomial vector field of $n$th degree. Let $H(n)$ denote the maximum number of limit cycles of a general planar polynomial vector field of degree $n$. As mentioned in $[1,2]$, there are little studies on an upper bound of $H(n)$, but there are many results on the lower bounds of $H(n)$; for example, $H(3) \geq 13, H(4) \geq 21$, and $H(5) \geq 28$ (see [3-7] for more details). For the hardness of Hilbert's 16th Problem, according to Smale [8], it might be appropriate to deal with Hilbert's 16th Problem restricted to the classical polynomial Liénard equations. In 2007, Dumortier et al. [9] found 4 limit cycles in a singular perturbed Liénard equation of degree 7 by applying geometric singular perturbation theory, and this result overturns Lin de Melo and Pugh's conjecture (see $[9,10]$ for details). The general form of planar singular perturbed differential equation can be given by as follows:

$$
\begin{gathered}
\varepsilon \frac{d x}{d \tau}=f(x, y, \lambda, \varepsilon), \\
\frac{d y}{d \tau}=g(x, y, \lambda, \varepsilon),
\end{gathered}
$$

where $f(x, y, \lambda)$ and $g(x, y, \lambda)$ are two smooth functions with respect to variables $(x, y, \lambda) \in \mathbb{R}^{2} \times \mathbb{R}^{k}$ and $\varepsilon$ is a small real number. As $\varepsilon>0$ and small, we make the time scaling $t=$ $\tau / \varepsilon$ and get the following equivalent standard form of slowfast system which has the same phase portraits as the one of system (1) with the slow variable $x$ and the fast variable $y$ :

$$
\begin{aligned}
& \frac{d x}{d t}=f(x, y, \lambda), \\
& \frac{d y}{d t}=\varepsilon g(x, y, \lambda) .
\end{aligned}
$$

Let $\varepsilon=0$ in system (1) and (2), we, respectively, obtain the following limiting system (3) and (4)

$$
\begin{gathered}
f(x, y, \lambda)=0, \\
\dot{y}=g(x, y, \lambda), \\
\dot{x}=f(x, y, \lambda), \\
\dot{y}=0 .
\end{gathered}
$$

System (3) and (4) are, respectively, called the reduced equation and the layer equation of system (1). For problems of planar singular perturbed system (1), the reduced equation captures essentially the slow dynamics and the layer equation captures the fast dynamics. The layer equation is a onedimensional dynamical system in the fast variable $y$ with the slow variable $x$ acting as a parameter. 
The equation $f(x, y, \lambda)=0$ defines the critical manifold $S$ of the equilibrium of the layer equation (4). The reduced equation describes the dynamics on the critical manifold $S$. Due to geometric singular perturbation theory of Fenichel (see [11] for more details), normally hyperbolic pieces of critical manifolds turn to locally invariant slow manifolds for $\varepsilon$ sufficiently small. Hence under suitable assumptions, orbits of singular perturbed system (2) can be obtained as perturbations of a slow-fast orbit which consist of pieces of the reduced equation (3) and the layer equation (4). Slow-fast orbit is not orbit of system (2), and it is the limit set of system (2) as $\varepsilon$ approaches 0 .

At contact points that are isolated points on the critical manifold where normal hyperbolicity is lost, the blow-up method pioneered by Dumortier and Roussarie [12] is a powerful geometric tool in the analysis of orbits of system (2) near nonhyperbolic points. According to the paper [13], we know that the admitted contact points $(x, y, \lambda)=\left(x_{0}, y_{0}, \lambda_{0}\right)$ of system (2) have been divided into two classes: a generic jump point and a generic turning point. If, after translation, rescaling of the variables $(x, y)$, and rescaling of time, system (2) can, locally near $(0,0,0)$, be, respectively, written as

$$
\begin{gathered}
\dot{x}=y-x^{2}+x^{3} h_{1}(x, y, \varepsilon, \lambda), \\
\dot{y}=-\varepsilon\left(1+x h_{2}(x, y, \varepsilon, \lambda)\right),
\end{gathered}
$$

or

$$
\begin{gathered}
\dot{x}=y-x^{2}+x^{3} h_{1}(x, y, \varepsilon, \lambda), \\
\dot{y}=-\varepsilon\left(a(\lambda)+x+x^{2} h_{2}(x, y, \varepsilon, \lambda)\right),
\end{gathered}
$$

then correspondingly nonhyperbolic point $\left(x_{0}, y_{0}, \lambda_{0}\right)$ of system (2) is called a generic jump point or a generic turning point of system (2), where functions $h_{1}$ and $h_{2}$ are smooth and $a\left(\lambda_{0}\right)=0$ and $a(\lambda)$ is smooth at $\lambda_{0}$ in case of a generic turning point.

If a slow-fast orbit of system (2) denoted by $\Gamma$ is closed, then $\Gamma$ is called slow-fast cycle. Further a slow-fast cycle $\Gamma$ is called common (see [9]) if all its slow curves have the same type: attracting or repelling. A slow-fast cycle $\Gamma$ is called a canard cycle if it contains both attracting and repelling slow curves. Here slow-fast cycle is not periodic orbit of system (2), but a limit periodic set as $\varepsilon$ approaches 0 and the limit cycles that are close to slow-fast cycle are called relaxation oscillations. In 2007, Dumortier et al. [9] proved that at least three limit cycles of Liénard equations with wellchosen polynomial $f$ of degree 7 bifurcate from the canard cycle consisting of two jump points by analyzing the zeros of slow divergence integral of canard cycle (see Figure 1).

In 2011, de Maesschalck and Dumortier [10] showed that four limit cycles bifurcated from the canard cycle of a singular perturbed Liénard system of degree six, which consists of a single fast orbit and a single slow curve and contains one turning point (see Figure 2). It can be clearly checked that the slow curve on one side of the turning point is attracting, and the part on the other side is repelling. This kind of canard cycle is said to be a 1-breaking parameter family of canard cycle. The main method used is to study the zeros of slow divergence integral of canard cycle.

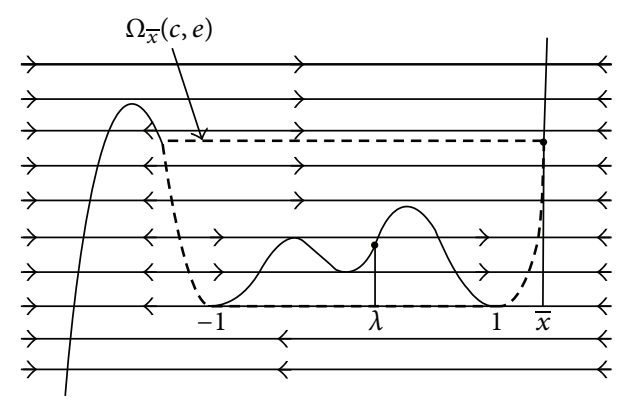

FIGURE 1: Canard cycle having two jump points.

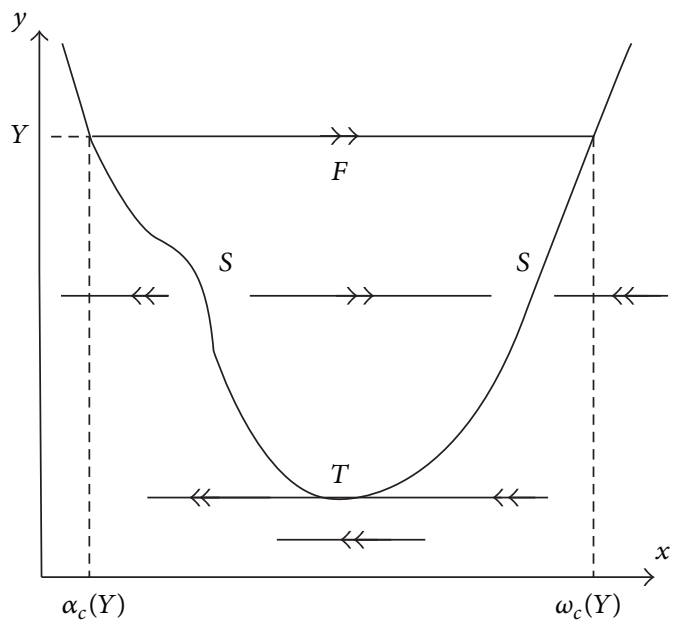

FIGURE 2: Canard cycle having one turning point.

In 2007, Dumortier and Roussarie [14, 15] considered two-dimensional slow-fast systems with a layer equation exhibiting canard cycle which contains a turning point and a fast orbit connecting two jump points (see Figure 3). At both the turning point and jump point, the presence of two parameters permitting generic breaking is assumed. The conditions of existing one (two or three) limit cycle in the above planar system are given by studying the fixed points of the Poincare map near canard cycles.

In this paper, we want to study the number of limit cycles near canard cycle which contains two turning points $p_{2}, p_{3}$ and two jump points $p_{1}, p_{4}$ that allow three breaking mechanisms and each one corresponds to one phase parameter (see Figure 4).

This paper is organized as follows. Our main results will be presented in the first part. The proofs of the results are presented in the second part. In the third part, a concrete example of planar singular Liénard equation existing three limit cycles will be given.

Consider the following smooth slow-fast Liénard system $X_{\lambda, \varepsilon}:$

$$
X_{\lambda, \varepsilon}:\left\{\begin{array}{l}
\dot{x}=y-F_{a}(x), \quad \lambda=(a, b, c) \in \mathbb{R}^{3}, \\
\dot{y}=\varepsilon g(x, b, c),
\end{array}\right.
$$

where $\lambda=(a, b, c)$ is near $(0,0,0)$. 


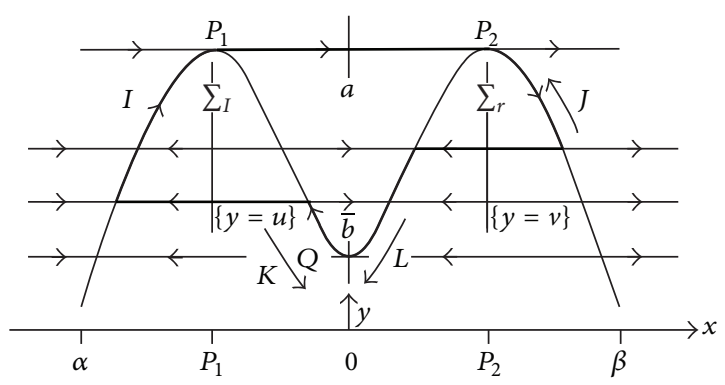

FIgURE 3: Canard cycles having two jump points and one turning point.

In the following, we assume that the smooth functions $F_{a}(x), g(x, b, c)$ fulfill the following conditions.

$\left(H_{1}\right)$ On the interval $\left(-x_{0}, x_{0}\right)$, as $a=0$, the function $F_{0}(x)$ has five singular points: two Morse maximum points at $p_{1}, p_{4}$, two Morse minimum points at $p_{2}, p_{3}$, and one Morse maximum at $p_{0}$ with $-x_{0}<p_{1}<p_{2}<$ $p_{0}<p_{3}<p_{4}<x_{0}$. The parameter $a$ is just the difference $a=F_{a}\left(p_{4}\right)-F_{a}\left(p_{1}\right)$. Let $\alpha_{0}=F_{0}(0)$, $\alpha_{1}=F_{0}\left(p_{2}\right), \alpha_{2}=F_{0}\left(p_{3}\right), \alpha=\max \left\{\alpha_{1}, \alpha_{2}\right\}$, and $\beta=F_{0}\left(p_{4}\right)=F_{0}\left(p_{1}\right)$. Then the values $F_{0}\left( \pm x_{0}\right)$ are assumed to be below the minimum value $\min \left\{\alpha_{1}, \alpha_{2}\right\}$.

$\left(H_{2}\right)$ Suppose that $g(0,0,0)=0, g\left(0,0, p_{2}\right)=0, g(0,0$, $\left.p_{3}\right)=0, g\left(0,0, p_{1}\right) \neq 0$, and $g\left(0,0, p_{4}\right) \neq 0$, but $\partial g(0$, $\left.0, p_{i}\right) / \partial x \neq 0, i=2,3, \partial g\left(0,0, p_{2}\right) / \partial b \neq 0$ and $\partial g(0,0$, $\left.p_{3}\right) / \partial c \neq 0, g(0,0, x)>0$ for $p_{1}<x<p_{2}$ or $p_{0}<x<$ $p_{3}, g(0,0, x)<0$ for $p_{2}<x<p_{0}$ or $p_{3}<x<p_{4}$ (see Figure 4).

$\left(H_{3}\right)$ When $\lambda=(0,0,0)$, there exists a canard cycle containing four horizontal segments: one between the two Morse maxima $x=p_{1}, x=p_{4}$ denoted by $\sigma_{h}$, one below the left Morse maximum value and at the height $y=u$ denoted by $\sigma_{l}$, one below the right Morse maximum value and at the height $y=v$ denoted by $\sigma_{r}(v)$, and one at the height $y=w$ and between $p_{2}$ and $p_{3}$ denoted by $\sigma_{m}$, where $u \in\left(\alpha_{1}, \beta\right), v \in\left(\alpha_{2}, \beta\right)$, $w \in\left(\alpha, \alpha_{0}\right)$, and the corresponding canard cycle is denoted by $\Gamma_{u v w}$.

An essential tool to study the limit cycles bifurcated from the canard cycle is the slow divergence integral (see $[9,10,13-$ $15]$ ); the slow divergence integral of the slow curve of system (7) between $x_{1}, x_{2}$ is defined as follows:

$$
\operatorname{Int}\left(x_{1}, x_{2}\right)=-\int_{x_{1}}^{x_{2}} \frac{1}{g(x, 0,0)}\left(\frac{d F_{0}(x)}{d x}\right)^{2} d x \text {. }
$$

Consider canard cycle $\Gamma_{u v w}$ of system (7). Let $x_{1}(u), x_{2}(u)$, respectively, denote $x$-coordinates of intersection points between $\sigma_{l}$ and slow curve $y=F_{0}(x)$, where $-x_{0}<$ $x_{2}(u)<x_{1}(u)<p_{2}$; let $x_{1}(w), x_{2}(w)$, respectively, denote $x$ coordinates of intersection points between $\sigma_{m}$ and $y=F_{0}(x)$, where $p_{2}<x_{1}(w)<x_{2}(w)<p_{3}$; let $x_{1}(v), x_{2}(v)$, respectively, denote $x$-coordinates of intersection points between $\sigma_{r}$ and curve $y=F_{0}(x)$, where $p_{3}<x_{1}(v)<x_{2}(v)<x_{0}$. By

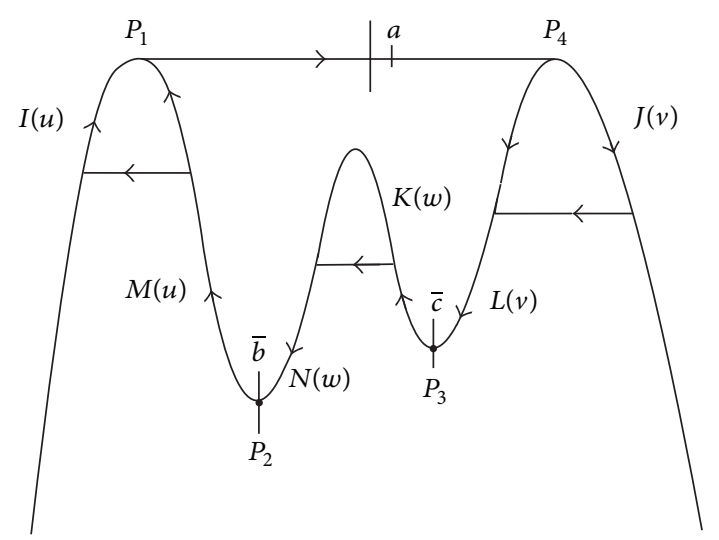

FIGURE 4: Canard cycle having two jump points and two turning points.

applying the slow divergence integral formula introduced in (8) to canard cycle $\Gamma_{u v w}$ of system (7), we get the following six integrals:

$$
\begin{array}{cl}
I(u)=\operatorname{Int}\left(x_{2}(u), p_{1}\right), & M(u)=\operatorname{Int}\left(x_{1}(u), p_{2}\right), \\
N(w)=\operatorname{Int}\left(x_{1}(w), p_{2}\right), & K(w)=\operatorname{Int}\left(x_{2}(w), p_{3}\right), \\
L(v)=\operatorname{Int}\left(x_{1}(v), p_{3}\right), & J(v)=\operatorname{Int}\left(x_{2}(v), p_{4}\right) .
\end{array}
$$

It is easy to check that

$$
\begin{gathered}
M^{\prime}(u), L^{\prime}(v), N^{\prime}(w), K^{\prime}(w)<0, \\
I^{\prime}(u), J^{\prime}(v)>0, \quad u \in\left(\alpha_{1}, \beta\right), \\
v \in\left(\alpha_{2}, \beta\right), \quad w \in\left(\alpha, \alpha_{0}\right) .
\end{gathered}
$$

Then, the canard cycle $\Gamma_{u v w}$ is associated with the above six functions: $I(u), J(v), K(w), L(v), M(u)$, and $N(w)$, which are the slow divergence integrals of six slow curves contained in $\Gamma_{u v w}$. In detail, two of these curves are located on the left of $p_{2}$ and their slow divergence integrals are functions of $u$, two of them are on the right of $p_{3}$ and their slow divergence integrals are functions of $v$, and two of them are between $p_{2}$ and $p_{3}$ and their slow divergence integrals are functions of $w$ (see Figure 4).

Now we give the following main results.

Theorem 1. Consider a general slow-fast Liénard system (7). Suppose that the smooth functions $F_{a}(x), g(x, b, c)$ fulfill the conditions $H_{1}, H_{2}, H_{3}$. Let $D(u, v, w)=J(v)-I(u)+M(u)-$ $N(w)+K(w)-L(v)$ denote the total slow divergence integral of $\Gamma_{\text {uvw }}$ of system (7).

(1) If there exist $u_{0} \in\left(\alpha_{1}, \beta\right), v_{0} \in\left(\alpha_{2}, \beta\right)$, and $w_{0} \in$ $\left(\alpha, \alpha_{0}\right)$ such that $D\left(u_{0}, v_{0}, w_{0}\right) \neq 0$, then for $\varepsilon>0$ and small enough $\left(u_{0}, v_{0}, w_{0}\right)$ is a regular point of $\Phi_{\varepsilon}$, whose explicit expression will be given in Section 2 and system (7) has a hyperbolic relaxation oscillation which is near $\Gamma_{u v w}$. 
(2) If there exist $u_{0} \in\left(\alpha_{1}, \beta\right), v_{0} \in\left(\alpha_{2}, \beta\right)$, and $w_{0} \in\left(\alpha, \alpha_{0}\right)$ such that $I\left(u_{0}\right)-J\left(v_{0}\right)>0, M\left(u_{0}\right)-N\left(w_{0}\right)=0$, and $I\left(u_{0}\right)-J\left(v_{0}\right)=K\left(w_{0}\right)-L\left(v_{0}\right)$, then for $\varepsilon>0$ and small enough there exits $\left(u_{0}(\varepsilon), v_{0}(\varepsilon), w_{0}(\varepsilon)\right)$ that is a generic fold singularity of $\Phi_{\varepsilon}$. A relaxation oscillation bifurcates from $\Gamma_{u v w}$ and this semistable limit cycle is generically unfolded by the parameter $(a, b, c)$ for $\varepsilon>0$ and small enough, producing a pair of hyperbolic limit cycles of system (7).

(3) If there exist $u_{0} \in\left(\alpha_{1}, \beta\right), v_{0} \in\left(\alpha_{2}, \beta\right)$, and $w_{0} \in$ $\left(\alpha, \alpha_{0}\right)$ such that $J\left(v_{0}\right)-I\left(u_{0}\right)=0, M\left(u_{0}\right)-N\left(w_{0}\right)=$ $0, L\left(v_{0}\right)-K\left(w_{0}\right)=0$, and $J^{\prime}\left(v_{0}\right) M^{\prime}\left(u_{0}\right) K^{\prime}\left(w_{0}\right)-$ $I^{\prime}\left(u_{0}\right) L^{\prime}\left(v_{0}\right) N^{\prime}\left(w_{0}\right) \neq 0$, then for $\varepsilon>0$ and small enough there exits $\left(\widehat{u}_{0}(\varepsilon), \widehat{v}_{0}(\varepsilon), \widehat{w}_{0}(\varepsilon)\right)$ that is a generic cusp singularity of $\Phi_{\varepsilon}$. A codimension 2 relaxation oscillation bifurcates from $\Gamma_{u v w}$ and this degenerated limit cycle is generically unfolded by the parameter $(a, b, c)$ for $\varepsilon>0$ and small enough, producing system (7) having three hyperbolic limit cycles in the vicinity of canard cycle $\Gamma_{u v w}$.

\section{The Proof of Main Results}

To study the limit cycle of the $X_{\lambda, \varepsilon}$ near the canard cycle $\Gamma_{u v w}$, we choose one vertical section $C_{1}$ at $x=0$, cutting the segment $\sigma_{h}$, section $C_{2}$ transversal to the turning point $p_{2}$, and section $C_{3}$ transversal to the turning point $p_{3}$. The parameter $a$ is the breaking parameter for the section $C_{1}$, a rescaling of $b$, given by $\bar{b}=\varepsilon^{-1 / 2} b$, is the breaking parameter at $C_{2}$, and a rescaling of $c$, given by $\bar{c}=\varepsilon^{-1 / 2} c$, is the breaking parameter at $C_{3}$ (see [13] for more details). In the following, we denote $(a, \bar{b}, \bar{c})$ by $\bar{\lambda}$. Let $\sum_{l}, \sum_{m}$, and $\sum_{r}$ be three sections which are transverse to the horizontal segments $\sigma_{l}, \sigma_{m}, \sigma_{r}$, parameterized, respectively, by $u, v, w$.

To study the fixed points of the obtained Poincare map, first we give the following definition of $\varepsilon$-regularly smooth function and the following lemma by introducing the relationship between intermediate variables $u, v, w$ and $a, \bar{b}, \bar{c}$.

Definition 2 (see $[9,13])$. A function $f(z, \varepsilon)$, with $z \in \mathbb{R}^{p}$ for some $p \in \mathbb{N}$, is called $\varepsilon$-regularly smooth in $z$, if $f$ is continuous and all partial derivatives of $f$ with respect to $z$ exist and are continuous in $(z, \varepsilon)$.

Lemma 3. For $\varepsilon>0$ small enough, a limit cycle of system (7) cuts $\sum_{l}$ in $u, \sum_{m}$ in $w$, and $\sum_{r}$ in $v$ if and only if $(a, \bar{b}, \bar{c})=$ $\Phi_{\varepsilon}(u, v, w)$, where $\Phi_{\varepsilon}$ is given by the following:

$$
\Phi_{\varepsilon}:\left\{\begin{array}{l}
a=\exp \left(\frac{\bar{I}(u, \varepsilon)}{\varepsilon}\right)-\exp \left(\frac{\bar{J}(v, \varepsilon)}{\varepsilon}\right) \\
\bar{b}=\exp \left(\frac{\bar{M}(u, \varepsilon)}{\varepsilon}\right)-\exp \left(\frac{\bar{N}(w, \varepsilon)}{\varepsilon}\right) \\
\bar{c}=\exp \left(\frac{\bar{K}(w, \varepsilon)}{\varepsilon}\right)-\exp \left(\frac{\bar{L}(v, \varepsilon)}{\varepsilon}\right)
\end{array}\right.
$$

where $\bar{I}(u, \varepsilon), \bar{J}(v, \varepsilon), \bar{K}(w, \varepsilon), \bar{L}(v, \varepsilon), \bar{M}(u, \varepsilon)$, and $\bar{N}(w, \varepsilon)$ are $\varepsilon$-regularly smooth in $u, v, w$, and, respectively, equal to $I(u)$, $J(v), K(w), L(v), M(u)$, and $N(w)$ for $\varepsilon=0$.

Proof. From [13-15], due to the chosen orientation on the $C_{i}(i=1,2,3)$, the transitions have the following expressions:

(1) from $\sum_{l}$ to $C_{1}: u \rightarrow \exp (\bar{I}(u, \bar{\lambda}, \varepsilon) / \varepsilon)+f_{l}(\bar{\lambda}, \varepsilon)$,

(2) from $\sum_{l}$ to $C_{2}: u \rightarrow \exp (\bar{M}(u, \bar{\lambda}, \varepsilon) / \varepsilon)+g_{l}(\bar{\lambda}, \varepsilon)$,

(3) from $\sum_{m}$ to $C_{2}: w \rightarrow \exp (\bar{N}(w, \bar{\lambda}, \varepsilon) / \varepsilon)+h_{m_{1}}(\bar{\lambda}, \varepsilon)$,

(4) from $\sum_{m}$ to $C_{3}: w \rightarrow \exp (\bar{K}(w, \bar{\lambda}, \varepsilon) / \varepsilon)+h_{m_{2}}(\bar{\lambda}, \varepsilon)$,

(5) from $\sum_{r}$ to $C_{1}: v \rightarrow \exp (\bar{J}(v, \bar{\lambda}, \varepsilon) / \varepsilon)+f_{r}(\bar{\lambda}, \varepsilon)$,

(6) from $\sum_{r}$ to $C_{3}: v \rightarrow \exp (\bar{L}(v, \bar{\lambda}, \varepsilon) / \varepsilon)+g_{r}(\bar{\lambda}, \varepsilon)$,

where functions $f_{l}, g_{l}, f_{r}, g_{r}, h_{m_{1}}, h_{m_{2}}$ are $\varepsilon$-regularly smooth in $\bar{\lambda}$, and functions $\bar{I}(u, \bar{\lambda}, \varepsilon), \bar{K}(w, \bar{\lambda}, \varepsilon), \bar{L}(v, \bar{\lambda}, \varepsilon), \bar{M}(u, \bar{\lambda}, \varepsilon)$, $\bar{N}(w, \bar{\lambda}, \varepsilon), \bar{J}(v, \bar{\lambda}, \varepsilon)$ are $\varepsilon$-regularly smooth in $\bar{\lambda}$ and satisfy that $\bar{I}(u, 0,0)=I(u), \bar{K}(w, 0,0)=K(w), \bar{L}(v, 0,0)=L(v)$, $\bar{M}(u, 0,0)=M(u), \bar{J}(v, 0,0)=J(v)$, and $\bar{N}(w, 0,0)=N(w)$.

By using the same analysis as $[14,15]$, we get that the system of equations for the existence of limit cycles of system (7) is

$$
\begin{array}{r}
\exp \left(\frac{\bar{I}(u, \bar{\lambda}, \varepsilon)}{\varepsilon}\right)-\exp \left(\frac{\bar{J}(v, \bar{\lambda}, \varepsilon)}{\varepsilon}\right) \\
=f_{r}(\bar{\lambda}, \varepsilon)-f_{l}(\bar{\lambda}, \varepsilon)=a F(\bar{\lambda}, \varepsilon), \\
\exp \left(\frac{\bar{M}(u, \bar{\lambda}, \varepsilon)}{\varepsilon}\right)-\exp \left(\frac{\bar{N}(w, \bar{\lambda}, \varepsilon)}{\varepsilon}\right) \\
=h_{m_{1}}(\bar{\lambda}, \varepsilon)-g_{l}(\bar{\lambda}, \varepsilon)=\bar{b} H(\bar{\lambda}, \varepsilon), \\
\exp \left(\frac{\bar{K}(w, \bar{\lambda}, \varepsilon)}{\varepsilon}\right)-\exp \left(\frac{\bar{L}(v, \bar{\lambda}, \varepsilon)}{\varepsilon}\right) \\
=g_{r}(\bar{\lambda}, \varepsilon)-h_{m_{2}}(\bar{\lambda}, \varepsilon)=\bar{c} G(\bar{\lambda}, \varepsilon),
\end{array}
$$

where $F(\bar{\lambda}, 0) \neq 0, G(\bar{\lambda}, 0) \neq 0$, and $H(\bar{\lambda}, 0) \neq 0$. Rewrite the above equations into the following form:

$$
\begin{aligned}
& \exp \left(\frac{\bar{I}(u, \bar{\lambda}, \varepsilon)}{\varepsilon}\right)-\exp \left(\frac{\bar{J}(v, \bar{\lambda}, \varepsilon)}{\varepsilon}\right)=a, \\
& \exp \left(\frac{\bar{M}(u, \bar{\lambda}, \varepsilon)}{\varepsilon}\right)-\exp \left(\frac{\bar{N}(w, \bar{\lambda}, \varepsilon)}{\varepsilon}\right)=\bar{b}, \\
& \exp \left(\frac{\bar{K}(w, \bar{\lambda}, \varepsilon)}{\varepsilon}\right)-\exp \left(\frac{\bar{L}(v, \bar{\lambda}, \varepsilon)}{\varepsilon}\right)=\bar{c},
\end{aligned}
$$

where new functions $\bar{I}, \bar{J}, \bar{K}, \bar{L}, \bar{M}$, and $\bar{N}$ differ from the previous ones in terms of order $o(\varepsilon)$ and are $\varepsilon$-regularly smooth in $(u, v, w, \bar{\lambda})$. 
We can solve this system in $a, \bar{b}$, and $\bar{c}$ because the partial derivations of the left hand term with respect to $a, \bar{b}$, and $\bar{c}$ are flat in $\varepsilon$. So one can solve (13) to obtain $a=a(u, v, w, \varepsilon)$, $\bar{b}=b(u, v, w, \varepsilon)$, and $\bar{c}=c(u, v, w, \varepsilon)$, such that the functions $a(u, v, w, \varepsilon), \bar{b}(u, v, w, \varepsilon)$, and $\bar{c}(u, v, w, \varepsilon)$ have the same form as the left hand term of (13) except that the functions $\bar{I}, \bar{J}, \bar{K}, \bar{L}$, $\bar{M}, \bar{N}$ are replaced by new functions not depending on $\bar{\lambda}$ and which are $\varepsilon$-flat perturbations of the previous ones. We will continue to call them $\bar{I}(u, \varepsilon), \bar{J}(v, \varepsilon), \bar{K}(w, \varepsilon), \bar{L}(v, \varepsilon), \bar{M}(u, \varepsilon)$, $\bar{N}(w, \varepsilon)$, and $\bar{I}(u, 0)=I(u), \bar{J}(v, 0)=J(v), \bar{K}(w, 0)=K(w)$, $\bar{L}(v, 0)=L(v), \bar{M}(u, 0)=M(u), \bar{N}(w, 0)=N(w)$.

The proof of Lemma 3 is completed.

2.1. The Proof of the First Part of Theorem 1. We take $\varepsilon$ small enough and we view $\Phi_{\varepsilon}$ as a map from $(u, v, w)$ to $(a, \bar{b}, \bar{c})$. First by direct computation, we get the Jacobian matrix of map $\Phi_{\varepsilon}$ as follows:

$$
\frac{\partial \Phi_{\varepsilon}(u, v, w)}{\partial(u, v, w)}=\left(\begin{array}{ccc}
\frac{\bar{I}^{\prime} e^{\bar{I} / \varepsilon}}{\varepsilon} & -\frac{\bar{J}^{\prime} e^{\bar{J} / \varepsilon}}{\varepsilon} & 0 \\
\frac{\bar{M}^{\prime} e^{\bar{M} / \varepsilon}}{\varepsilon} & 0 & -\frac{\bar{N}^{\prime} e^{\bar{N} / \varepsilon}}{\varepsilon} \\
0 & -\frac{\bar{L}^{\prime} e^{\bar{L} / \varepsilon}}{\varepsilon} & \frac{\bar{K}^{\prime} e^{\bar{K} / \varepsilon}}{\varepsilon}
\end{array}\right),
$$

where $\bar{I}^{\prime}, \bar{M}^{\prime}$ are the partial derivatives with respect to $u, \bar{J}^{\prime}$, $\bar{L}^{\prime}$ are the partial derivatives with respect to $v$, and $\bar{K}^{\prime}, \bar{M}^{\prime}$ are the partial derivatives with respect to $w$.

Let $\Delta(u, v, w, \varepsilon)=\operatorname{det}\left(\partial \Phi_{\varepsilon}(u, v, w) / \partial(u, v, w)\right)$. To find the singular points of map $\Phi_{\varepsilon}$, by direct computation we get the following formula about the determinant $\Delta(u, v, w, \varepsilon)$ :

$$
\Delta=\frac{1}{\varepsilon^{3}}\left(e^{(\bar{J}+\bar{K}+\bar{M}) / \varepsilon} \bar{J}^{\prime} \bar{K}^{\prime} \bar{M}^{\prime}-e^{(\bar{I}+\bar{L}+\bar{N}) / \varepsilon} \bar{I}^{\prime} \bar{L}^{\prime} \bar{N}^{\prime}\right) .
$$

Let $\bar{D}=\bar{J}-\bar{I}+\bar{K}-\bar{L}+\bar{M}-\bar{N}$; then $D(u, v, w)=\bar{D}(u, v, w, 0)$ is exactly the complete slow divergence integral computed along the canard cycle $\Gamma_{u v w}$. With the function $\bar{D}$, we can rewrite $\Delta$ into

$$
\Delta=\frac{1}{\varepsilon^{3}} \bar{J}^{\prime} \bar{K}^{\prime} \bar{M}^{\prime} e^{(\bar{I}+\bar{L}+\bar{N}) / \varepsilon}\left(e^{\bar{D} / \varepsilon}-\frac{\bar{I}^{\prime} \bar{L}^{\prime} \bar{N}^{\prime}}{\bar{J}^{\prime} \bar{K}^{\prime} \bar{M}^{\prime}}\right) .
$$

For $\varepsilon>0$ and small, the equation for the singular points of the map $\Phi_{\varepsilon}$ given by $\Delta(u, v, w, \varepsilon)=0$ is equivalent to the equation

$$
E(u, v, w, \varepsilon)=\bar{D}(u, v, w, \varepsilon)-\varepsilon \log \frac{\bar{I}^{\prime} \bar{L}^{\prime} \bar{N}^{\prime}}{\bar{J}^{\prime} \bar{K}^{\prime} \bar{M}^{\prime}}=0 .
$$

It follows from (10) that the term in the logarithm in (17) is strictly positive. Under the conditions of the first part of Theorem 1, we can get that $\bar{D} \neq 0$ as $\varepsilon>0$ and small enough by noting $\varepsilon$-regularity of function $\bar{D}(u, v, w, \varepsilon)$. That means that the map $\Phi_{\varepsilon}$ is nondegenerated at the point $\left(u_{0}, v_{0}, w_{0}\right)$, so from Lemma 3, we get that system (7) has one limit cycle near $\Gamma_{u_{0} v_{0} \omega_{0}}$.

So the conclusion of the first part of Theorem 1 follows.

2.2. The Proof of the Second Part of Theorem 1. In this subsection, we give the proof of the second part of Theorem 1 .

First, we present the following lemma.

Lemma 4. As $\varepsilon>0$ and small enough, if there exists $P_{0}(\varepsilon)=$ $\left(u_{0}(\varepsilon), v_{0}(\varepsilon), w_{0}(\varepsilon)\right)$ that satisfies $\Delta(u, v, w, \varepsilon)=0$, then it holds that

$$
\left.\operatorname{grad} \Delta\right|_{P_{0}(\varepsilon)}=\left.k \cdot \operatorname{grad} E\right|_{P_{0}(\varepsilon)},
$$

where grad $\Delta=(\partial \Delta / \partial u, \partial \Delta / \partial v, \partial \Delta / \partial w)$ is the gradient of function $\Delta(u, v, w, \varepsilon)$ and $k$ is nonzero function at $\left(P_{0}(\varepsilon), \varepsilon\right)$.

Proof. From (10) in Section 1, we get that equation $E(u$, $v, w, \varepsilon)=0$ determines a surface $S_{1}$ in the neighborhood of point $P_{0}\left(u_{0}, v_{0}, w_{0}\right)$ for $\varepsilon>0$ and small. From the fact that $\Delta(u, v, w, \varepsilon)=0$ is equivalent to $E(u, v, w, \varepsilon)=0$, we get that the equation $\Delta(u, v, w, \varepsilon)=0$ also determines the surface $S_{1}$. For grad $\left.\Delta\right|_{P_{0}(\varepsilon)},\left.\operatorname{grad} E\right|_{P_{0}(\varepsilon)}$ are both the normal vectors of surface $S_{1}$ at the point $P_{0}(\varepsilon)$, then we get (18).

The proof of Lemma 4 is completed.

Proof of the Second Part of Theorem 1. First, we introduce functions $\varphi(u, v, \varepsilon), \psi(u, v, \varepsilon), \phi(u, v, \varepsilon)$ as follows:

$$
\Phi_{\varepsilon}:\left\{\begin{aligned}
a & =\exp \left(\frac{\bar{I}(u, \varepsilon)}{\varepsilon}\right)-\exp \left(\frac{\bar{J}(v, \varepsilon)}{\varepsilon}\right) \\
& \equiv \varphi(u, v, \varepsilon), \\
\bar{b} & =\exp \left(\frac{\bar{M}(u, \varepsilon)}{\varepsilon}\right)-\exp \left(\frac{\bar{N}(w, \varepsilon)}{\varepsilon}\right) \\
& \equiv \psi(u, w, \varepsilon), \\
\bar{c} & =\exp \left(\frac{\bar{K}(w, \varepsilon)}{\varepsilon}\right)-\exp \left(\frac{\bar{L}(v, \varepsilon)}{\varepsilon}\right) \\
& \equiv \phi(v, w, \varepsilon),
\end{aligned}\right.
$$

where the map $\Phi_{\varepsilon}$ is given in Lemma 3.

Denote $\left(u_{0}, v_{0}, w_{0}\right)$ by $P_{0}$, from assumptions in the second part of Theorem 1; then we get $E\left(P_{0}, 0\right)=0$ and $(\partial E / \partial u$. $\partial E / \partial v)\left.\right|_{\left(P_{0}, 0\right)} \neq 0$. For $\varepsilon>0$ and small, by applying Implicit Function Theorem, we can find surface $\widetilde{S}: v=v(u, w, \varepsilon)$ which satisfies the equation $E(u, v, w, \varepsilon)=0$. On surface $\widetilde{S}$, we choose point $\left(u_{0}(\varepsilon), v_{0}(\varepsilon), w_{0}(\varepsilon)\right)$ denoted by $P_{0}(\varepsilon)$ such that $E\left(P_{0}(\varepsilon), \varepsilon\right)=0, P_{0}(0)=P_{0}$; that is, $\Delta\left(P_{0}(\varepsilon), \varepsilon\right)=0$.

From $\left.(\partial \varphi / \partial v)\right|_{\left(P_{0}, 0\right)} \neq 0$ and Implicit Function Theorem, we can get $v=v(a, u, \varepsilon)$ from the first equation of (19). Also, from $\left.(\partial \phi / \partial w)\right|_{\left(P_{0}, 0\right)} \neq 0$, we can get $w=w(\bar{c}, v, \varepsilon)$ from the third one. Substituting $v=v(a, u, \varepsilon), w=w(\bar{c}, v, \varepsilon)$ into the second one, we get $\bar{b}=\psi(u, w(\bar{c}, v, \varepsilon), \varepsilon) \equiv \bar{\psi}(u, a, \bar{c}, \varepsilon)$. 
Next, we compute the first derivative of $\bar{b}$ with respect to $u$ as

$$
\begin{aligned}
\frac{d \bar{b}}{d u} & =\psi_{u}+\psi_{w} \frac{d w}{d u}=\psi_{u}+\psi_{w} \frac{\partial w}{\partial v} \frac{\partial v}{\partial u} \\
& =\psi_{u}+\psi_{w}\left(-\frac{\phi_{v}}{\phi_{w}}\right)\left(-\frac{\varphi_{u}}{\varphi_{v}}\right)=-\frac{\Delta}{\phi_{w} \varphi_{v}}
\end{aligned}
$$

Then, as $\Delta(u, v, w, \varepsilon)=0$ we compute the second derivative of $\bar{b}$ with respect to $u$ as

$$
\left.\frac{d^{2} \bar{b}}{d u^{2}}\right|_{\Delta=0}=\left.\frac{d}{d u}\left(\frac{-\Delta}{\phi_{w} \varphi_{v}}\right)\right|_{\Delta=0}=-\frac{d \Delta / d u}{\phi_{w} \varphi_{v}} .
$$

From (10), we get that $\left.(\partial D / \partial u)\right|_{P_{0}}=M^{\prime}\left(u_{0}\right)-I^{\prime}\left(u_{0}\right)<0$, $\left.(\partial D / \partial v)\right|_{P_{0}}=J^{\prime}\left(v_{0}\right)-L^{\prime}\left(v_{0}\right)>0$, and $\left.(\partial D / \partial w)\right|_{P_{0}}=K^{\prime}\left(w_{0}\right)-$ $N^{\prime}\left(w_{0}\right)$. So $\left.(\partial E / \partial u)\right|_{\left(P_{0}(\varepsilon), \varepsilon\right)}=\left.(\partial D / \partial u)\right|_{P_{0}}+O(\varepsilon)<0,(\partial E /$ $\partial v)\left.\right|_{\left(P_{0}(\varepsilon), \varepsilon\right)}=\left.(\partial D / \partial v)\right|_{P_{0}}+O(\varepsilon)>0$, and $\left.(\partial E / \partial w)\right|_{\left(P_{0}(\varepsilon), \varepsilon\right)}=$ $\left.(\partial D / \partial w)\right|_{P_{0}}+O(\varepsilon)$.

From Lemma 4, we get grad $\left.\Delta\right|_{\left(P_{0}(\varepsilon), \varepsilon\right)}=k\left(\left.(\partial D / \partial u)\right|_{P_{0}}+\right.$ $\left.O(\varepsilon),\left.(\partial D / \partial v)\right|_{P_{0}}+O(\varepsilon),\left.(\partial D / \partial w)\right|_{P_{0}}+O(\varepsilon)\right)$.

From (19), we get that

$$
\begin{gathered}
\frac{d v}{d u}=\frac{\bar{I}^{\prime}(u, \varepsilon)}{\bar{J}^{\prime}(v, \varepsilon)} e^{(\bar{I}(u, \varepsilon)-\bar{J}(v, \varepsilon)) / \varepsilon}>0, \\
\frac{d w}{d u}=\frac{\bar{M}^{\prime}(u, \varepsilon)}{\bar{N}^{\prime}(w, \varepsilon)} e^{(\bar{M}(u, \varepsilon)-\bar{N}(w, \varepsilon)) / \varepsilon}>0 .
\end{gathered}
$$

Noticing that $I\left(u_{0}\right)-J\left(v_{0}\right)>0, M\left(u_{0}\right)-N\left(w_{0}\right)=0$, we get that $e^{(\bar{I}(u, \varepsilon)-\bar{J}(v, \varepsilon)) / \varepsilon} \rightarrow+\infty, \varepsilon \rightarrow 0^{+}$and $e^{(\bar{M}(u, \varepsilon)-\bar{N}(w, \varepsilon)) / \varepsilon}=$ $O(1), \varepsilon \rightarrow 0^{+}$.

Then for $\varepsilon>0$ and small enough we get that

$$
\begin{aligned}
\left.\frac{d \Delta}{d u}\right|_{\left(P_{0}(\varepsilon), \varepsilon\right)} & =\left.\left(\frac{\partial \Delta}{\partial u}+\frac{\partial \Delta}{\partial v} \frac{d v}{d u}+\frac{\partial \Delta}{\partial w} \frac{d w}{d u}\right)\right|_{\left(P_{0}(\varepsilon), \varepsilon\right)} \\
& =\left.\left.\operatorname{grad} \Delta\right|_{\left(P_{0}(\varepsilon), \varepsilon\right)} \cdot\left(1, \frac{d v}{d u}, \frac{d w}{d u}\right)\right|_{\left(P_{0}(\varepsilon), \varepsilon\right)} \neq 0
\end{aligned}
$$

Because $\phi_{w} \varphi_{v} \neq 0,\left.\left(d^{2} \bar{b} / d u^{2}\right)\right|_{\left.\left(P_{0}(\varepsilon), \varepsilon\right)\right)} \neq 0$ is equivalent to $\left.(d \Delta / d u)\right|_{\left.\left(P_{0}(\varepsilon), \varepsilon\right)\right)} \neq 0$. So we get that $\left(u_{0}(\varepsilon), v_{0}(\varepsilon), w_{0}(\varepsilon)\right)$ is a fold point of map $\Phi_{\varepsilon}$. So from Lemma 3 and fold bifurcation of scalar map $\bar{b}=\bar{\psi}(u, a, \bar{c}, \varepsilon)$ (see [16]), we get that system (7) has two limit cycles near $\Gamma_{u v w}$ by unfolding the parameters $a$, $b, c$.

The conclusion of the second part of Theorem 1 follows.

2.3. The Proof of the Third Part of Theorem 1. In this subsection, we give the proof of the third part of Theorem 1. First, we present the following lemma.

Lemma 5. As $\varepsilon>0$ and small enough, if there exists $\widehat{P}_{0}(\varepsilon)=$ $\left(u_{0}(\varepsilon), v_{0}\left(u_{0}, \varepsilon\right), w_{0}\left(u_{0}, v_{0}, \varepsilon\right)\right)$ that satisfies $\Delta(u, v, w, \varepsilon)=0$, $d \Delta(u, v, w, \varepsilon) / d u=0$, and $e^{(\bar{I}-\bar{J}) / \varepsilon}=e^{(\bar{M}-\bar{N}) / \varepsilon}$, then it holds that

$$
\left.\operatorname{grad}\left(\frac{d \Delta}{d u}\right)\right|_{\widehat{P}_{0}(\varepsilon)}=\left.\widehat{k} \cdot \operatorname{grad} \widehat{E}\right|_{\widehat{P}_{0}(\varepsilon)},
$$

where $\widehat{E}(u, v, w, \varepsilon)=\bar{I}(u, \varepsilon)-\bar{J}(v, \varepsilon)-\varepsilon \ln \left(-\bar{J}^{\prime} \bar{N}^{\prime}(\partial D / \partial u) /\right.$ $\left.\left(\bar{I}^{\prime} \bar{N}^{\prime}(\partial D / \partial v)+\bar{J}^{\prime} \bar{M}^{\prime}(\partial D / \partial w)\right)+O(\varepsilon)\right), \operatorname{grad} \widehat{E}=(\partial \widehat{E} / \partial u$, $\partial \widehat{E} / \partial v, \partial \widehat{E} / \partial w)$ is the gradient of function $\widehat{E}(u, v, w, \varepsilon)$, and $\widehat{k}$ is nonzero function at $\left(\widehat{P}_{0}(\varepsilon), \varepsilon\right)$.

Proof. From the proof of the second part of Theorem 1, as $\Delta(u, v, w, \varepsilon)=0$ we get

$$
\begin{aligned}
\frac{d \Delta}{d u}=k\left[\frac{\bar{I}^{\prime}}{\bar{J}^{\prime}} e^{(\bar{I}-\bar{J}) \varepsilon}\left(\frac{\partial D}{\partial v}+O(\varepsilon)\right)\right. \\
\left.+\frac{\bar{M}^{\prime}}{\bar{N}^{\prime}} e^{(\bar{M}-\bar{N}) / \varepsilon}\left(\frac{\partial D}{\partial w}+O(\varepsilon)\right)+\left(\frac{\partial D}{\partial u}+O(\varepsilon)\right)\right] .
\end{aligned}
$$

From $e^{(\bar{I}-\bar{J}) / \varepsilon}=e^{(\bar{M}-\bar{N}) / \varepsilon}$, we get that $d \Delta / d u=0$ is equivalent to

$$
e^{(\bar{I}-\bar{J}) / \varepsilon}=\frac{-(\partial D / \partial u) \bar{J}^{\prime} \bar{N}^{\prime}}{\bar{I}^{\prime} \bar{N}^{\prime}(\partial D / \partial v)+\bar{J}^{\prime} \bar{M}^{\prime}(\partial D / \partial w)}+O(\varepsilon) .
$$

So rewrite (26), and we get that the equation $d \Delta / d u=0$ is equivalent to $\widehat{E}(u, v, w, \varepsilon)=0$ under assumptions $\Delta=0$, $e^{(\bar{I}-\bar{J}) / \varepsilon}=e^{(\bar{M}-\bar{N}) / \varepsilon}$ for $\varepsilon>0$ and small. That means that both equations $d \Delta(u, v, w, \varepsilon) / d u=0$ and $\widehat{E}(u, v, w, \varepsilon)=$ 0 determine the same surface $\widehat{S}$ in the neighborhood of $\widehat{P}_{0}(\varepsilon)$. For $\left.\operatorname{grad}(d \Delta / d u)\right|_{\widehat{P}_{0}(\varepsilon)},\left.\operatorname{grad} \widehat{E}\right|_{\widehat{P}_{0}(\varepsilon)}$ are both the normal vectors of surface $\widehat{S}$ at the point $\widehat{P}_{0}(\varepsilon)$, then we get (24).

The proof of Lemma 5 is completed.

Proof of the Third Part of Theorem 1. From Lemmas 4 and 5, we get that

$$
\begin{gathered}
\Delta(u, v, w, \varepsilon)=0, \\
\frac{d \Delta(u, v, w, \varepsilon)}{d u}=0
\end{gathered}
$$

is equivalent to

$$
\begin{aligned}
& E(u, v, w, \varepsilon)=0, \\
& \widehat{E}(u, v, w, \varepsilon)=0 .
\end{aligned}
$$

It is easy to check that $E\left(u_{0}, v_{0}, w_{0}, 0\right)=0, \widehat{E}\left(u_{0}, v_{0}\right.$, $\left.w_{0}, 0\right)=0$ and surfaces $S_{1}: E(u, v, w, \varepsilon)=0$ and $S_{2}: \widehat{E}(u$, $v, w, \varepsilon)=0$ transversely intersect along a curve $l_{\varepsilon}$ which passes through point $\left(u_{0}, v_{0}, w_{0}, 0\right)$. Therefore, for $\varepsilon>0$ and small enough, we choose point $\widehat{P}_{0}(\varepsilon)=$ $\left(u_{0}(\varepsilon), v_{0}\left(u_{0}, \varepsilon\right), w_{0}\left(u_{0}, v_{0}, \varepsilon\right)\right)$ on the curve $l_{\varepsilon}$. Then $\Delta\left(\widehat{P}_{0}(\varepsilon)\right.$, $\varepsilon)=0,\left.(d \Delta / d u)\right|_{\left(\widehat{P}_{0}(\varepsilon), \varepsilon\right)}=0$. 
Consider the map $\Phi_{\varepsilon}$ given in (19). By applying a similar process to the one in the proof the second part of Theorem 1 , we get $\bar{b}=\bar{\psi}(u, a, c, \varepsilon), d \bar{b} / d u=-\Delta / \phi_{w} \varphi_{v}$, and $\left(d^{2} \bar{b} /\right.$ $\left.d u^{2}\right)\left.\right|_{\Delta=0}=-(d \Delta / d u) / \phi_{w} \varphi_{v}$. Next, under the assumptions given in the third part of Theorem 1, we compute the third derivative of $\bar{b}$ with respect to $u$ at $\widehat{P}_{0}(\varepsilon)$ and get

$$
\begin{aligned}
\left.\frac{d^{3} \bar{b}}{d u^{3}}\right|_{\widehat{P}_{0}(\varepsilon)} & =-\left.\frac{(d / d u)(d \Delta / d u)}{\phi_{w} \varphi_{v}}\right|_{\widehat{P}_{0}(\varepsilon)} \\
& =\left.\frac{-1}{\phi_{w} \varphi_{v}} \cdot \operatorname{grad}\left(\frac{d \Delta}{d u}\right)\right|_{\widehat{P}_{0}(\varepsilon)} \cdot\left(1, \frac{d v}{d u}, \frac{d w}{d u}\right)_{\widehat{P}_{0}(\varepsilon)} .
\end{aligned}
$$

From Lemma 5, we get $\left.\operatorname{grad}(d \Delta / d u)\right|_{\hat{P}_{0}(\varepsilon)}=\widehat{k} \cdot\left(I^{\prime}\left(u_{0}\right)+\right.$ $\left.O(\varepsilon), J^{\prime}\left(v_{0}\right)+O(\varepsilon), O(\varepsilon)\right)$.

From (19) and (26), we get that

$$
\begin{aligned}
\left.\frac{d v}{d u}\right|_{\widehat{P}_{0}(\varepsilon)} & =\left[\frac{\bar{I}^{\prime}(u, \varepsilon)}{\bar{J}^{\prime}(v, \varepsilon)} e^{(\bar{I}(u, \varepsilon)-\bar{J}(v, \varepsilon)) / \varepsilon}\right]_{\widehat{P}_{0}(\varepsilon)} \\
& =\left[\frac{-I^{\prime} N^{\prime}(\partial D / \partial u)}{\bar{I}^{\prime} N^{\prime}(\partial D / \partial v)+J^{\prime} M^{\prime}(\partial D / \partial w)}\right]_{P_{0}}+O(\varepsilon), \\
\left.\frac{d w}{d u}\right|_{\widehat{P}_{0}(\varepsilon)} & =\left[\frac{\bar{M}^{\prime}(u, \varepsilon)}{\bar{N}^{\prime}(w, \varepsilon)} e^{(\bar{M}(u, \varepsilon)-\bar{N}(w, \varepsilon)) / \varepsilon}\right]_{\hat{P}_{0}(\varepsilon)} \\
& =\left[\frac{-J^{\prime} M^{\prime}(\partial D / \partial u)}{I^{\prime} N^{\prime}(\partial D / \partial v)+J^{\prime} M^{\prime}(\partial D / \partial w)}\right]_{P_{0}}+O(\varepsilon) .
\end{aligned}
$$

Then for $\varepsilon>0$ and small enough we get that

$$
\begin{aligned}
& \left.\frac{d^{2} \Delta}{d u^{2}}\right|_{\widehat{P}_{0}(\varepsilon)} \\
& =\widehat{k} \cdot\left(I^{\prime}\left(u_{0}\right)+J^{\prime}\left(v_{0}\right)\right. \\
& \left.\quad \times\left[\frac{-I^{\prime} N^{\prime}(\partial D / \partial u)}{I^{\prime} N^{\prime}(\partial D / \partial v)+J^{\prime} M^{\prime}(\partial D / \partial w)}\right]_{P_{0}}+O(\varepsilon)\right) \\
& =I^{\prime}\left(u_{0}\right) \widehat{k} \cdot\left[\frac{J^{\prime} K^{\prime} M^{\prime}-I^{\prime} L^{\prime} N^{\prime}}{I^{\prime} N^{\prime}(\partial D / \partial v)+J^{\prime} M^{\prime}(\partial D / \partial w)}+O(\varepsilon)\right]_{P_{0}}
\end{aligned}
$$$$
\neq 0 \text {. }
$$

For $\left.\left(\widehat{k} / \phi_{w} \varphi_{v}\right)\right|_{\widehat{P}_{0}(\varepsilon)} \neq 0,\left.\left(d^{3} \bar{b} / d u^{3}\right)\right|_{\widehat{P}_{0}(\varepsilon)} \neq 0$ is equivalent to $\left.\left(d^{2} \Delta / d u^{2}\right)\right|_{\widehat{P}_{0}(\varepsilon)} \neq 0$; then we get that $\widehat{P}_{0}(\varepsilon)$ is a cusp point of map $\bar{b}=\bar{\psi}(u, a, \bar{c}, \varepsilon)$ for $\varepsilon>0$ and small enough. From Lemma 3 and cusp bifurcation of scalar map [16], we get that the above degenerated limit cycle is generically unfolded by the parameters $(a, b, c)$, for $\varepsilon>0$ and small enough, producing system (7) having three hyperbolic limit cycles near $\Gamma_{u v w}$.

The proof of the third part of Theorem 1 is completed.

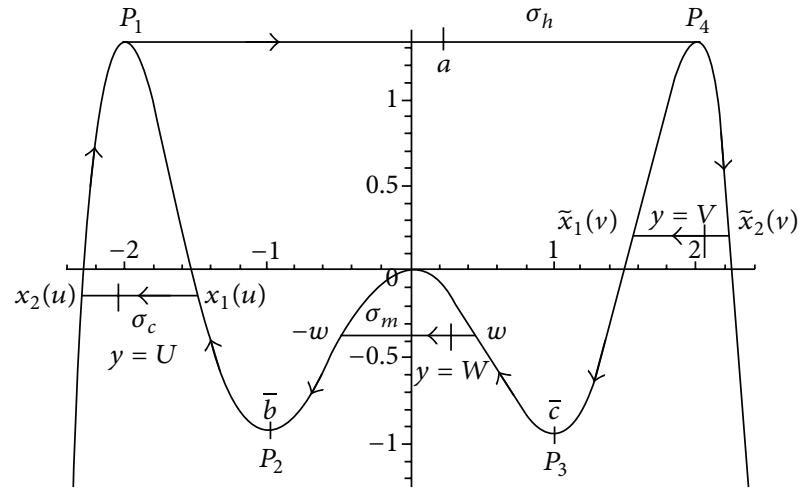

Figure 5: Canard cycles $\Gamma_{U V W}$ of system (32).

\section{Application to Polynomial Liénard Equation}

In this section, we will apply Theorem 1 to the following polynomial Liénard equation:

$$
X_{a, b, c, \varepsilon}:\left\{\begin{aligned}
\dot{x}= & y-\left(-\frac{1}{6} x^{6}+\frac{5}{4} x^{4}-2 x^{2}+a x\right), \\
\dot{y}= & \varepsilon(b-(x-1) \\
& \left.\times\left(1+c_{1}(x-1)+c_{2}(x-1)^{3}\right)\right) \\
& \times(c-(x+1))(-x) .
\end{aligned}\right.
$$

For this system, $F_{a}(x)=\left(-(1 / 6) x^{6}+(5 / 4) x^{4}-2 x^{2}+a x\right)$. Here we use symbols $p_{i}, i=1,2,3,4$ defined in Section 2. By direct computation we get that $p_{1}=-2, p_{4}=2$; the values of $F_{0}(x)$ at these two points are both $4 / 3$ and the values of $F_{0}(x)$ at the points $p_{2}=-1, p_{3}=1$ are both $-11 / 12$ and $F_{0}(0)=0$.

For a given $V \in(-11 / 12,0)$, the equation $F_{0}(x)=$ $V$ will have three roots on the right side of the $y$-axis. For the roots, the formula in the above equation contains complex number, so we choose the smallest one of the above three roots and denote it by the variable $v$, where $v \in$ $(0,1)$. By noting that relation between $u$ and $v$ is one to one relation, we express the other two roots in the form $\tilde{x}_{1}(v)=(1 / 2)\left(\sqrt{15-2 v^{2}-\sqrt{3} \sqrt{11+20 v^{2}-4 v^{4}}}\right), \tilde{x}_{2}(v)=$ $(1 / 2)\left(\sqrt{\left.15-2 v^{2}+\sqrt{3} \sqrt{11+20 v^{2}-4 v^{4}}\right)}\right.$, where $v<\tilde{x}_{1}(v)<$ $\tilde{x}_{2}(v), v \in(0,1)$. Using the same way, we take $y=U$, $U \in(-11 / 12,0)$; then the equation $F_{0}(x)=U$ will have three roots on the left side of the $y$-axis; we choose the biggest one of the above three roots and denote it by the variable $u$; we express the other two roots in the form $x_{1}(u)=-\widetilde{x}_{1}(u)$, $x_{2}(u)=-\tilde{x}_{2}(u)$ and $x_{2}(u)<x_{1}(u)<u, u \in(-1,0)$. Also $F_{0}(x)=y$ is symmetrical about 0 , so the straight line $y=W, W \in(-11 / 12,0)$, between -1 and 1 will intersect the curve with two points; then we, respectively, denote their $x$-coordinate by $-w, w$, where $w \in(0,1)$. The corresponding canard cycle $\Gamma_{U V W}$ can be seen in Figure 5.

Let $P(x)=(1 / 8) x^{8}-(3 / 2) x^{6}+6 x^{4}-8 x^{2}, Q_{1}(x)=$ $-(1 / 9) x^{9}+(1 / 8) x^{8}+(9 / 7) x^{7}-(24 / 5) x^{5}-(3 / 2) x^{6}+6 x^{4}+$ $(16 / 3) x^{3}-8 x^{2}, Q_{2}(x)=-(1 / 11) x^{11}+(3 / 10) x^{10}+(2 / 3) x^{9}-$ $(13 / 4) x^{8}+(3 / 7) x^{7}+(21 / 2) x^{6}-(56 / 5) x^{5}-6 x^{4}+16 x^{3}-8 x^{2}$; then 
from the slow divergence integral formula (8) in Section 2, we get that

$$
\begin{aligned}
& M(u)=P(-1)-P\left(-\tilde{x}_{1}(u)\right) \\
& +c_{1}\left(Q_{1}(-1)-Q_{1}\left(-\tilde{x}_{1}(u)\right)\right) \\
& +c_{2}\left(Q_{2}(-1)-Q_{2}\left(-\tilde{x}_{1}(u)\right)\right) \\
& +O\left(\left(\left|c_{1}\right|+\left|c_{2}\right|\right)^{2}\right), \\
& N(w)=P(-1)-P(-w) \\
& +c_{1}\left(Q_{1}(-1)-Q_{1}(-w)\right) \\
& +c_{2}\left(Q_{2}(-1)-Q_{2}(-w)\right) \\
& +O\left(\left(\left|c_{1}\right|+\left|c_{2}\right|\right)^{2}\right), \\
& I(u)=P(-2)-P\left(-\tilde{x}_{2}(u)\right) \\
& +c_{1}\left(Q_{1}(-2)-Q_{1}\left(-\tilde{x}_{2}(u)\right)\right) \\
& +c_{2}\left(Q_{2}(-2)-Q_{2}\left(-\tilde{x}_{2}(u)\right)\right) \\
& +O\left(\left(\left|c_{1}\right|+\left|c_{2}\right|\right)^{2}\right), \\
& J(v)=P(2)-P\left(\tilde{x}_{2}(v)\right) \\
& +c_{1}\left(Q_{1}(2)-Q_{1}\left(\tilde{x}_{2}(v)\right)\right) \\
& +c_{2}\left(Q_{2}(2)-Q_{2}\left(\tilde{x}_{2}(v)\right)\right) \\
& +O\left(\left(\left|c_{1}\right|+\left|c_{2}\right|\right)^{2}\right), \\
& L(v)=P(1)-P\left(\tilde{x}_{1}(v)\right) \\
& +c_{1}\left(Q_{1}(1)-Q_{1}\left(\tilde{x}_{1}(v)\right)\right) \\
& +c_{2}\left(Q_{2}(1)-Q_{2}\left(\tilde{x}_{1}(v)\right)\right) \\
& +O\left(\left(\left|c_{1}\right|+\left|c_{2}\right|\right)^{2}\right) \\
& K(w)=P(1)-P(w)+c_{1}\left(Q_{1}(1)-Q_{1}(w)\right) \\
& +c_{2}\left(Q_{2}(1)-Q_{2}(w)\right) \\
& +O\left(\left(\left|c_{1}\right|+\left|c_{2}\right|\right)^{2}\right) \text {. }
\end{aligned}
$$

Firstly, consider $M(u)-N(w)=0$; that is,

$$
\begin{aligned}
& P(-w)-P\left(-\tilde{x}_{1}(u)\right)+c_{1}\left(Q_{1}(-w)-Q_{1}\left(-\tilde{x}_{1}(u)\right)\right) \\
& \quad+c_{2}\left(Q_{2}(-w)-Q_{2}\left(-\tilde{x}_{1}(u)\right)\right)+O\left(\left(\left|c_{1}\right|+\left|c_{2}\right|\right)^{2}\right)=0 .
\end{aligned}
$$

When $c_{1}=c_{2}=0$, from the fact that the function $P(x)$ is monotone on the interval $(-2,-1)$ we get $w=\tilde{x}_{1}(u)$. Let $w=\tilde{x}_{1}(u)+c_{1} w_{1}(u)+c_{2} w_{2}(u)+O\left(\left(\left|c_{1}\right|+\left|c_{2}\right|\right)^{2}\right)$ and substitute it into (34); by setting the coefficients of $c_{1}$ and $c_{2}$ of obtained equation to equal zeros, we get $w_{1}(u)=0, w_{2}(u)=0$. Therefore, we get

$$
w(u)=\tilde{x}_{1}(u)+O\left(\left(\left|c_{1}\right|+\left|c_{2}\right|\right)^{2}\right)
$$

Secondly, consider $I(u)-J(v)=0$; that is,

$$
\begin{aligned}
& P\left(\tilde{x}_{2}(v)\right)-P\left(-\tilde{x}_{2}(u)\right) \\
& \quad+c_{1}\left(Q_{1}(-2)\right)-Q_{1}(2)+Q_{1}\left(\tilde{x}_{2}(v)\right) \\
& \quad-Q_{1}\left(-\tilde{x}_{2}(u)\right)+c_{2}\left(Q_{2}(-2)\right)-Q_{2}(2)+Q_{2}\left(\tilde{x}_{2}(v)\right) \\
& \quad-Q_{2}\left(-\tilde{x}_{2}(u)\right)+O\left(\left(\left|c_{1}\right|+\left|c_{2}\right|\right)^{2}\right)=0 .
\end{aligned}
$$

By noticing that the even function $P(x)$ is strictly monotone on the interval $(-2,-1)$ and $\tilde{x}_{2}(-u)=\tilde{x}_{2}(u)$, we get $v=-u$ as $c_{1}=c_{2}=0$.

Let

$$
v=-u+c_{1} v_{1}(u)+c_{2} v_{2}(u)+O\left(\left(\left|c_{1}\right|+\left|c_{2}\right|\right)^{2}\right),
$$

and put it into (36); then by setting the coefficients of $c_{1}$ and $c_{2}$ of obtained equation to equal zeros, we get

$$
\begin{aligned}
& v_{1}(u)=\frac{Q_{1}(2)-Q_{1}(-2)+Q_{1}\left(-\tilde{x}_{2}(u)\right)-Q_{1}\left(\tilde{x}_{2}(u)\right)}{P^{\prime}\left(\tilde{x}_{2}(-u)\right) \tilde{x}_{2}^{\prime}(-u)}, \\
& v_{2}(u)=\frac{Q_{2}(2)-Q_{2}(-2)+Q_{2}\left(-\tilde{x}_{2}(u)\right)-Q_{2}\left(\tilde{x}_{2}(u)\right)}{P^{\prime}\left(\tilde{x}_{2}(-u)\right) \tilde{x}_{2}^{\prime}(-u)} .
\end{aligned}
$$

Thirdly, consider function $L(v)-K(w)$; that is,

$$
\begin{aligned}
L(v)-K(w)= & P(w)-P\left(\tilde{x}_{1}(v)\right)+c_{1}\left(Q_{1}(w)\right) \\
& -Q_{1}\left(\tilde{x}_{1}(v)\right)+c_{2}\left(Q_{2}(w)-Q_{2}\left(\tilde{x}_{1}(v)\right)\right) \\
& +O\left(\left(\left|c_{1}\right|+\left|c_{2}\right|\right)^{2}\right) .
\end{aligned}
$$

Putting the expressions (35) and (37) into (39), we get

$$
L(v)-K(w)=c_{1} \zeta_{1}(u)+c_{2} \zeta_{2}(u)+O\left(\left|c_{1}\right|+\left|c_{2}\right|\right)^{2},
$$

where

$$
\begin{aligned}
\zeta_{1}(u)= & -P^{\prime}\left(\tilde{x}_{1}(-u)\right) \tilde{x}_{1}^{\prime}(-u) \\
& \times \frac{Q_{1}(2)-Q_{1}(-2)+Q_{1}\left(-\tilde{x}_{2}(u)\right)-Q_{1}\left(\tilde{x}_{2}(u)\right)}{P^{\prime}\left(\tilde{x}_{2}(-u)\right) \tilde{x}_{2}^{\prime}(-u)}, \\
\zeta_{2}(u)= & -P^{\prime}\left(\tilde{x}_{1}(-u)\right) \tilde{x}_{1}^{\prime}(-u) \\
& \times \frac{Q_{2}(2)-Q_{2}(-2)+Q_{2}\left(-\tilde{x}_{2}(u)\right)-Q_{2}\left(\tilde{x}_{2}(u)\right)}{P^{\prime}\left(\tilde{x}_{2}(-u)\right) \tilde{x}_{2}^{\prime}(-u)} .
\end{aligned}
$$

From the definition of function $Q_{i}(x), i=1,2$, we get that $\zeta_{1}(u) \cdot \zeta_{2}(u) \neq 0, u \in(-1,0)$. 


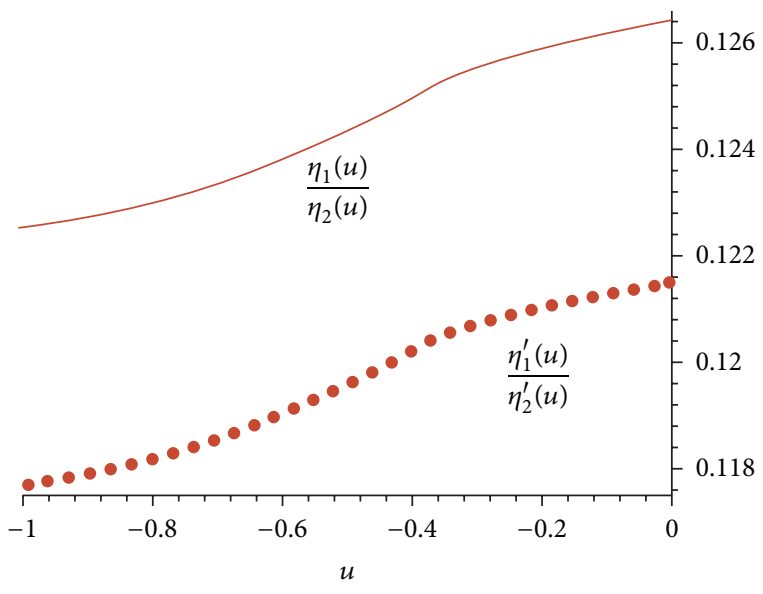

FIGURE 6: The graph of functions $\eta_{1}(u) / \eta_{2}(u)$ and $\eta_{1}^{\prime}(u) / \eta_{2}^{\prime}(u)$ on the interval $(-1,0)$ are, respectively, plotted in solid line and point line.

Denote $\eta_{1}(u)=Q_{1}(2)-Q_{1}(-2)+Q_{1}\left(-\widetilde{x}_{2}(u)\right)-Q_{1}\left(\widetilde{x}_{2}(u)\right)$, $\eta_{2}(u)=Q_{2}(2)-Q_{2}(-2)+Q_{2}\left(-\widetilde{x}_{2}(u)\right)-Q_{2}\left(\tilde{x}_{2}(u)\right)$ and denote

$$
\theta(u)=-\frac{P^{\prime}\left(\tilde{x}_{1}(-u)\right) \tilde{x}_{1}^{\prime}(-u)}{P^{\prime}\left(\widetilde{x}_{2}(-u)\right) \tilde{x}_{2}^{\prime}(-u)} .
$$

For $\theta(u) \neq 0, u \in(-1,0)$, then $\zeta_{1}(u) / \zeta_{2}(u)=\eta_{1}(u) / \eta_{2}(u)$.

Denote $H(u)=L(v(u))-K(w(u))$, where $w(u)$ and $v(u)$ are, respectively, implicitly determined by $M(u)-N(w)=0$ and $I(u)-J(v)=0$.

By direct computations, we get

$$
\begin{aligned}
\frac{d H(u)}{d u} & =L^{\prime} \cdot v^{\prime}(u)-K^{\prime} \cdot w^{\prime}(u) \\
& =L^{\prime} \cdot \frac{I^{\prime}}{J^{\prime}}-K^{\prime} \cdot \frac{M^{\prime}}{N^{\prime}}=\frac{I^{\prime} L^{\prime} N^{\prime}-J^{\prime} K^{\prime} M^{\prime}}{J^{\prime} N^{\prime}} .
\end{aligned}
$$

On the other hand from expressions (40), we get that

$$
\frac{d H(u)}{d u}=c_{1} \zeta_{1}^{\prime}(u)+c_{2} \zeta_{2}^{\prime}(u)+O\left(\left|c_{1}\right|+\left|c_{2}\right|\right)^{2} .
$$

As $H(u)=0$, then we get

$$
\frac{d H(u)}{d u}=c_{1} \theta(u) \eta_{1}^{\prime}(u)+c_{2} \theta(u) \eta_{2}^{\prime}(u)+O\left(\left|c_{1}\right|+\left|c_{2}\right|\right)^{2} .
$$

The graph of functions $\eta_{1}(u) / \eta_{2}(u)$ and $\eta_{1}^{\prime}(u) / \eta_{2}^{\prime}(u)$ on the interval $(-1,0)$ is plotted in Figure 6.

From the graph in Figure 6, we conclude that, for any $u_{0} \in(-1,0)$, there exists $c_{2}=-\left(\eta_{2}\left(u_{0}\right) / \eta_{1}\left(u_{0}\right)\right) c_{1}+O\left(c_{1}^{2}\right), 0<$ $\left|c_{1}\right| \ll 1$ such that equation $H(u)=0$ holds and $d H\left(u_{0}\right) / d u=$ $c_{1} \theta\left(u_{0}\right)\left(\eta_{1}^{\prime}\left(u_{0}\right)+\left(\eta_{1}\left(u_{0}\right) / \eta_{2}\left(u_{0}\right)\right) \eta_{2}^{\prime}\left(u_{0}\right)\right)+O\left(c_{1}^{2}\right) \neq 0$ for $c_{1}>0$ and small enough.

In other words, as $c_{2}=-\left(\eta_{2}\left(u_{0}\right) / \eta_{1}\left(u_{0}\right)\right) c_{1}+O\left(c_{1}^{2}\right)$, $0<\left|c_{1}\right| \ll 1$, it holds that $I^{\prime}\left(u_{0}\right) L^{\prime}\left(v_{0}\right) N^{\prime}\left(w_{0}\right)-$ $J^{\prime}\left(v_{0}\right) K^{\prime}\left(w_{0}\right) M^{\prime}\left(u_{0}\right) \neq 0$, where $w_{0}=w\left(u_{0}\right), v_{0}=v\left(u_{0}\right)$. So there exists $\left(u_{0}, v_{0}, w_{0}\right)$ which satisfies the conditions in the third part of Theorem 1, and from conclusions of Theorem 1, we get that system (32) has three hyperbolic relaxation oscillations near canard cycle $\Gamma_{U V W}$, where $U=$ $F_{0}\left(u_{0}\right), V=F_{0}\left(v\left(u_{0}\right)\right), W=F_{0}\left(w\left(u_{0}\right)\right), u_{0} \in(-1,0)$.

\section{Conflict of Interests}

The authors declare that there is no conflict of interests regarding the publication of this paper.

\section{Acknowledgments}

This project was supported by the National Natural Science Foundation of China (NSFC 11101189, NSFC 11171135), the Priority Academic Program Development of Jiangsu Higher Education Institutions (PAPD), and the Natural Science Foundation of Jiangsu Province of China (BK2012282).

\section{References}

[1] J. Li, "Hilbert's 16th problem and bifurcations of planar polynomial vector fields," International Journal of Bifurcation and Chaos in Applied Sciences and Engineering, vol. 13, no. 1, pp. 47106, 2003.

[2] M. Han and J. Li, "Lower bounds for the Hilbert number of polynomial systems," Journal of Differential Equations, vol. 252, no. 4, pp. 3278-3304, 2012.

[3] C. Li, C. Liu, and J. Yang, "A cubic system with thirteen limit cycles," Journal of Differential Equations, vol. 246, no. 9, pp. 3609-3619, 2009.

[4] J. Li and Y. Liu, "New results on the study of $Z_{q}$-equivariant planar polynomila vector fields," Qualitative Theory of Dynamical Systems, vol. 9, pp. 167-219, 2010.

[5] J. Yang, M. Han, J. Li, and P. Yu, "Existence conditions of thirteen limit cycles in a cubic system," International Journal of Bifurcation and Chaos, vol. 20, no. 8, pp. 2569-2577, 2010.

[6] Y. Wu, Y. Gao, and M. Han, "On the number and distributions of limit cycles in a quintic planar vector field," International Journal of Bifurcation and Chaos, vol. 18, no. 7, pp. 1939-1955, 2008.

[7] Y. Wu and M. Han, "On the number and distributions of limit cycles of a planar quarticvector field," International Journal of Bifurcation and Chaos, vol. 23, no. 4, 2013.

[8] S. Smale, "Mathematical problems for the next century," Mathematical Intelligencer, vol. 20, no. 2, pp. 7-15, 1998.

[9] F. Dumortier, D. Panazzolo, and R. Roussarie, "More limit cycles than expected in Liénard equations," Proceedings of the American Mathematical Society, vol. 135, no. 6, pp. 1895-1904, 2007.

[10] P. de Maesschalck and F. Dumortier, "Classical Lienard equations of degree $n \geq 6$ can have $[n-1 / 2]+2$ limit cycles," Journal of Differential Equations, vol. 250, no. 4, pp. 2162-2176, 2011.

[11] N. Fenichel, "Geometric singular perturbation theory for ordinary differential equations," Journal of Differential Equations, vol. 31, no. 1, pp. 53-98, 1979.

[12] F. Dumortier and R. Roussarie, Canard Cycles and Center Manifolds, vol. 577 of Memoirs of the American Mathematical Society, American Mathematical Society, 1996. 
[13] F. Dumortier, "Slow divergence integral and balanced canard solutions," Qualitative Theory of Dynamical Systems, vol. 10, no. 1, pp. 65-85, 2011.

[14] F. Dumortier and R. Roussarie, "Canard cycles with two breaking parameters," Discrete and Continuous Dynamical Systems, vol. 17, no. 4, pp. 787-806, 2007.

[15] F. Dumortier and R. Roussarie, "Bifurcation of relaxation oscillations in dimension two," Discrete and Continuous Dynamical Systems, vol. 19, no. 4, pp. 631-674, 2007.

[16] J. K. Hale and H. Kocak, Dynamics and Bifurcations, Spinger, New York, NY, USA, 1991. 


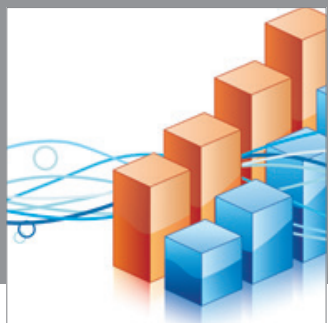

Advances in

Operations Research

mansans

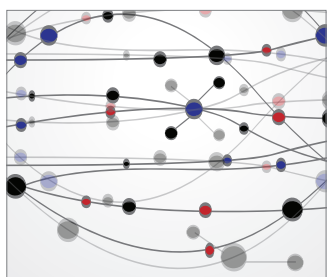

The Scientific World Journal
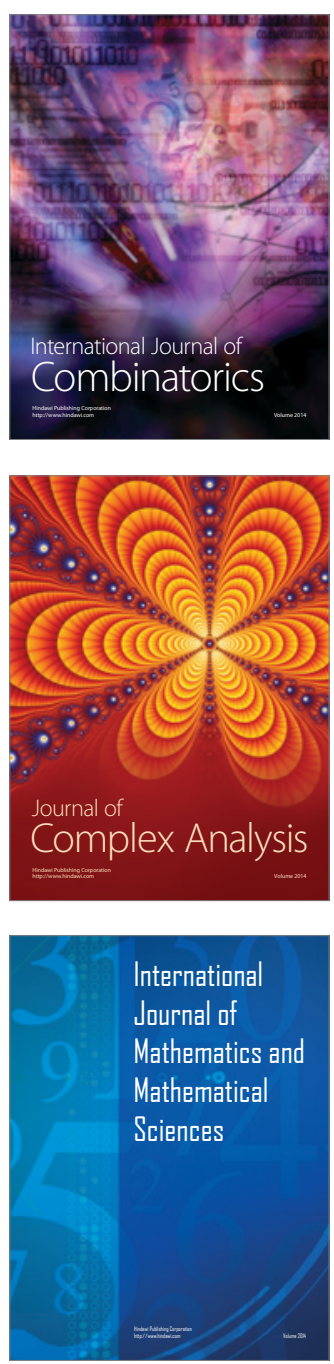
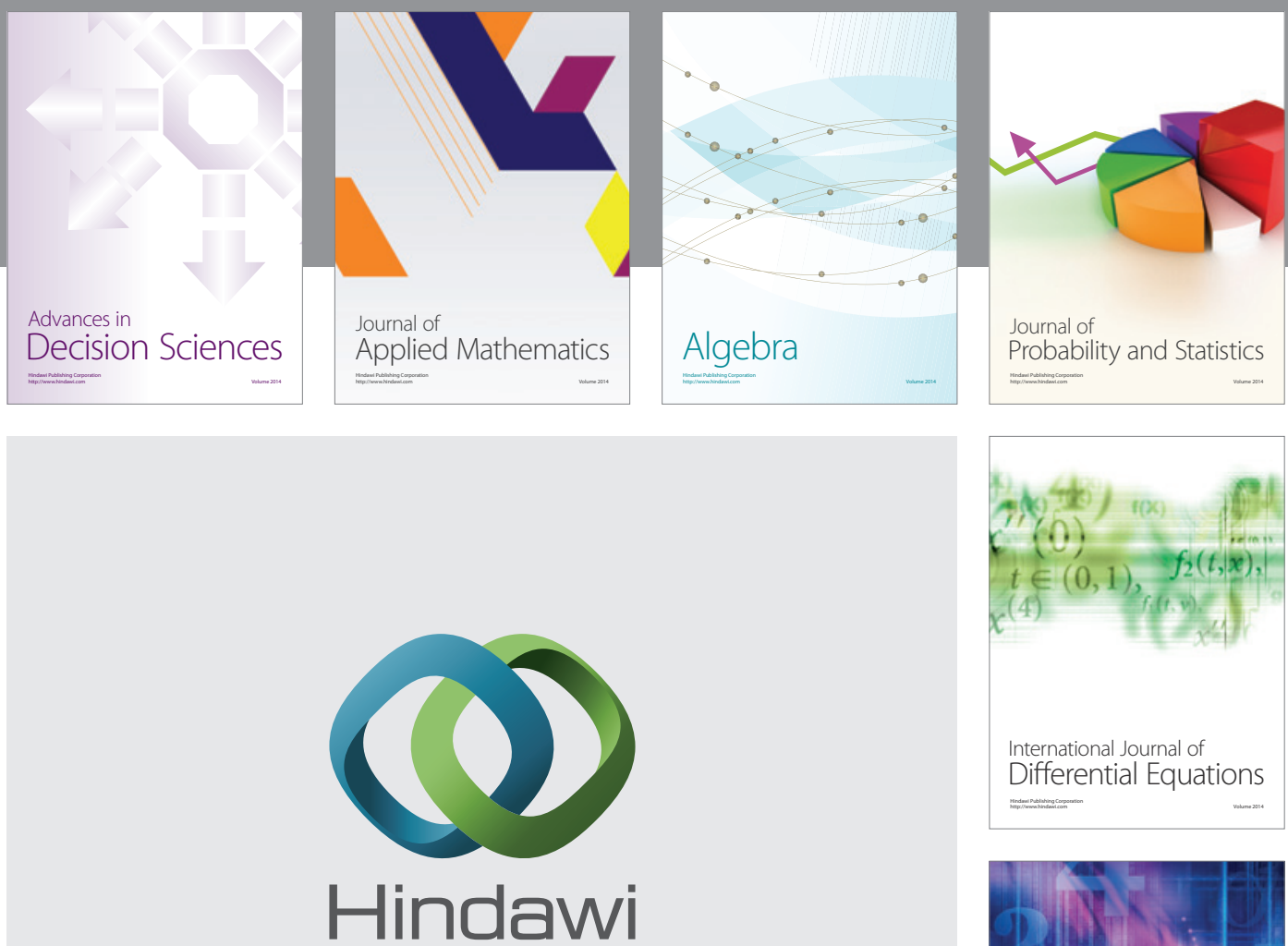

Submit your manuscripts at http://www.hindawi.com
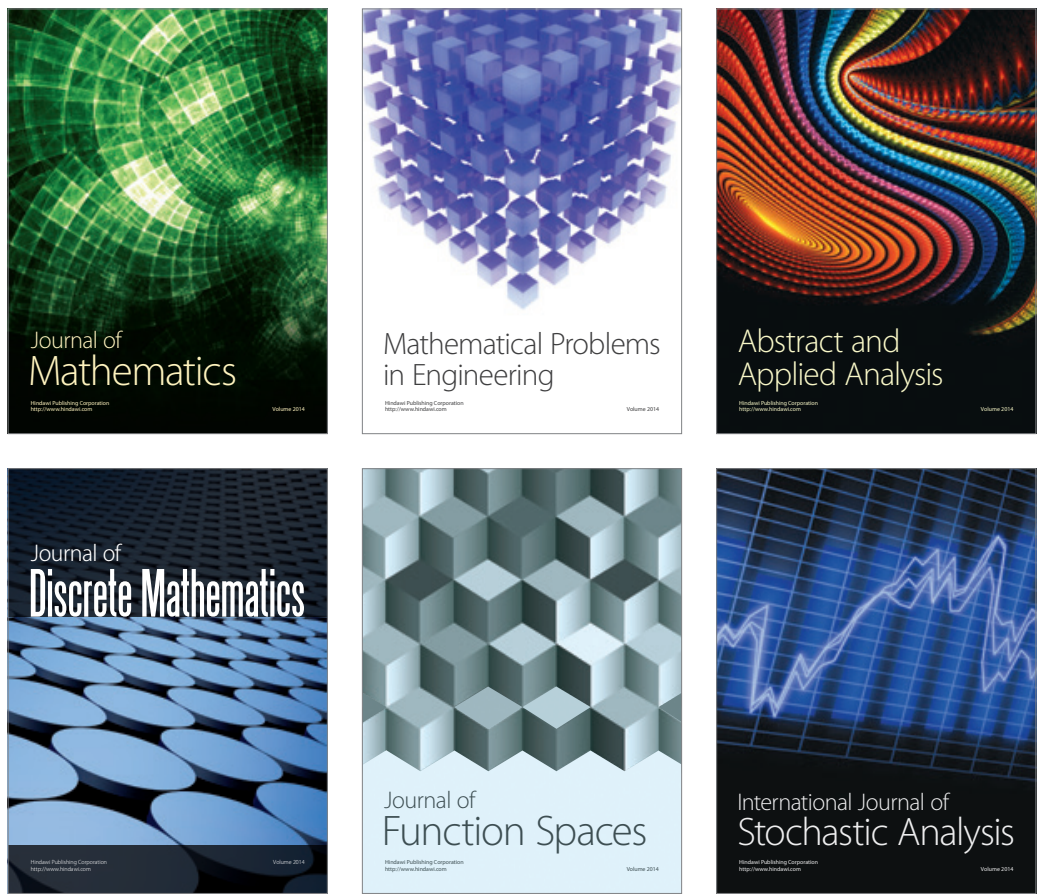

Journal of

Function Spaces

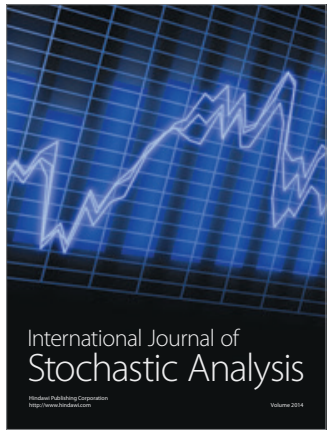

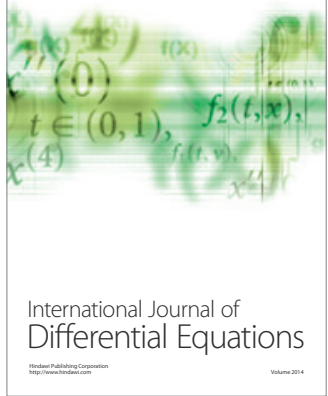
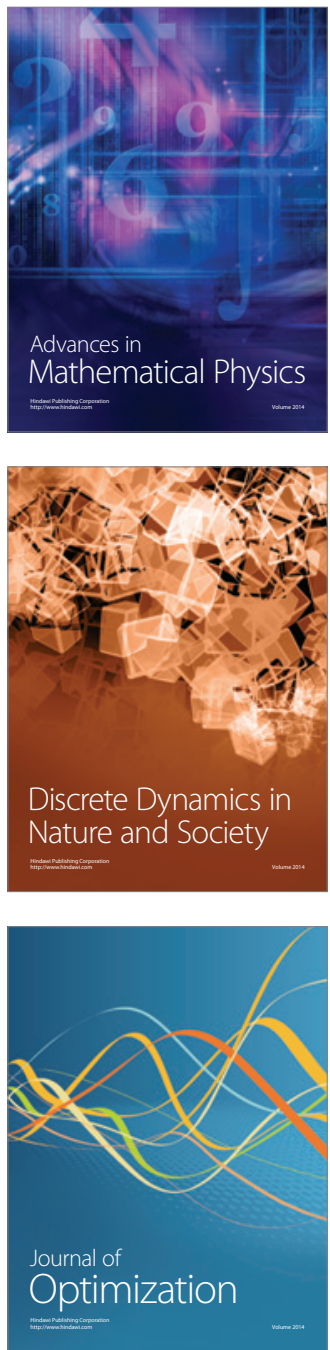